Virginia Commonwealth University VCU Scholars Compass

2009

\title{
Functionalized heterofullerenes for hydrogen storage
}

\author{
Qiang Sun \\ Peking University, Virginia Commonwealth University \\ Qian Wang \\ Virginia Commonwealth University \\ Puru Jena \\ Virginia Commonwealth University, pjena@vcu.edu
}

Follow this and additional works at: http://scholarscompass.vcu.edu/phys_pubs

Part of the Physics Commons

Sun, Q. Wang, Q. Jena, P. Functionalized heterofullerenes for hydrogen storage. Applied Physics Letters, 94, 013111 (2009). Copyright (C 2009 AIP Publishing LLC.

\section{Downloaded from}

http://scholarscompass.vcu.edu/phys_pubs/16 


\title{
Functionalized heterofullerenes for hydrogen storage
}

\author{
Qiang Sun, ${ }^{1,2, a)}$ Qian Wang, ${ }^{2}$ and Puru Jena ${ }^{2}$ \\ ${ }^{1}$ Department of Advanced Materials and Nanotechnology and Center for Applied Physics and Technology, \\ Peking University, Beijing 100871, China \\ ${ }^{2}$ Department of Physics, Virginia Commonwealth University, Richmond, Virginia 23284, USA
}

(Received 18 September 2008; accepted 3 December 2008; published online 9 January 2009)

\begin{abstract}
Using density functional theory, we show that $\mathrm{Li}$ decorated $\mathrm{B}$ doped heterofullerene $\left(\mathrm{Li}_{12} \mathrm{C}_{48} \mathrm{~B}_{12}\right)$ has the following desired properties of a hydrogen storage material. (1) The $\mathrm{Li}$ atoms remain isolated. (2) Through charge transfer to electron deficient $\mathrm{C}_{48} \mathrm{~B}_{12}$ heterofullerene, the $\mathrm{Li}$ atoms become positively charged. (3) Each $\mathrm{Li}$ atom is able to bind up to three $\mathrm{H}_{2}$ molecules, which remain in molecular form, and the binding energies of successive $\mathrm{H}_{2}$ molecules are in the range of $0.135-0.172 \mathrm{eV} / \mathrm{H}_{2}$, suitable for ambient temperature storage. (4) The gravimetric density reaches the 9 wt \% limit necessary for applications in the mobile industry. (C) 2009 American Institute of Physics. [DOI: 10.1063/1.3058678]
\end{abstract}

The limited supply of fossil fuels and their adverse effect on the environment due to the emissions of greenhouse gases and volatile organic chemicals have necessitated the search for alternative energy sources that are abundant, renewable, pollution-free, secure, and cost effective. In this regard hydrogen is being considered as a potential candidate. In addition to being the most abundant element in the universe, hydrogen offers many advantages over other fuels. It is nontoxic, clean to use, and packs more energy per unit mass than any other fuel. However, one of the most challenging problems in hydrogen economy is our ability to store hydrogen with large gravimetric and volumetric density at near ambient thermodynamic conditions. It is widely accepted that for technological applications, solid state materials are necessary for storing hydrogen. To meet 9 wt \% gravimetric density, storage materials should consist of elements lighter than aluminum, and for near ambient pressure and temperature applications, the binding energy of hydrogen should be in the order of $0.2 \mathrm{eV} / \mathrm{H}_{2}$. ${ }^{1}$ Since the energy with which hydrogen is bound in light materials is an order of magnitude higher than the above value, attention has focused on nanostructures of light elements, particularly carbon fullerenes and nanotubes. $^{2-8}$

It was recently proposed $^{7,8}$ that decorating carbon fullerenes and nanotubes with transition metal atoms can bind hydrogen in large quantities with binding energies in the ideal range for mobile applications. Later studies, ${ }^{9}$ however, showed that homogeneously coated $\mathrm{C}_{60}$ fullerenes with transition metal atoms are metastable and the transition metals would cluster on the fullerene surface, thus undermining their ability to store hydrogen in large quantities. It was suggested that one can overcome the clustering problem by decorating $\mathrm{C}_{60}$ with $\mathrm{Li}$ atoms, ${ }^{10}$ but the binding energy of $\mathrm{H}_{2}$ molecules became too low for room temperature applications. The central challenge has been to find metal atoms that will resist clustering and yet bind to hydrogen with binding energy intermediate between physisorption and chemisorption. In this letter we propose such material.

Using first principles calculation, we show that Li coated heterofullerene $\mathrm{C}_{48} \mathrm{~B}_{12}$ can overcome the difficulties outlined

${ }^{a)}$ Electronic mail: sunq@coe.pku.edu.cn. in the above. Note that it was demonstrated a long time ago ${ }^{11}$ that a $\mathrm{Li}^{+}$ion can bind to at least six $\mathrm{H}_{2}$ molecules with binding energies between 0.253 and $0.202 \mathrm{eV} / \mathrm{H}_{2}$. In $\mathrm{C}_{60} \mathrm{Li}$, the charge transfer from $\mathrm{Li}$ to $\mathrm{C}_{60}$, which has an electron affinity of $2.66 \mathrm{eV}$, does allow Li to remain in a nearly +1 charge state and hence bind to hydrogen with a binding energy of $0.18 \mathrm{eV} / \mathrm{H}_{2}$. However, as more $\mathrm{Li}$ atoms decorate the $\mathrm{C}_{60}$, the charge on each $\mathrm{Li}$ decreases and so does the binding energy of successive $\mathrm{H}_{2}$ molecules. We show that this situation can be avoided by initially doping $\mathrm{C}_{60}$ with $\mathrm{B}$. In $\mathrm{C}_{48} \mathrm{~B}_{12} \mathrm{Li}_{12}$, $\mathrm{Li}$ atoms not only remain isolated but also can each bind up to three $\mathrm{H}_{2}$ molecules with binding energies between 0.172 and $0.135 \mathrm{eV} / \mathrm{H}_{2}$, leading to a gravimetric density of 9 wt $\%$, suitable for ambient temperature storage. $^{1}$

The chemistry of $\mathrm{C}_{60-n} \mathrm{~B}_{n}$ clusters is governed by their electron deficient character. Past calculations ${ }^{12}$ showed that the electron affinities of $\mathrm{C}_{60-n} \mathrm{~B}_{n}$ clusters $(n=1-12)$ are larger than that of $\mathrm{C}_{60}$ and behave as electron acceptors. Thus, it is expected that when $\mathrm{Li}$ atoms decorate the fullerene surface, charge transfer to electron deficient $\mathrm{C}_{60-n} \mathrm{~B}_{n}$ clusters may leave them in a more positively charged state than that in $\mathrm{C}_{60}$. We have studied the equilibrium stability and geometry of $\mathrm{Li}_{12} \mathrm{C}_{48} \mathrm{~B}_{12}$ cluster and its ability to adsorb hydrogen using density functional theory and generalized gradient approximation for exchange and correlation. We used a super cell approach where the cluster was surrounded by $15 \AA$ of vacuum space along $x, y$, and $z$ directions. The $\Gamma$ point was used to represent the Brillouin zone due to the large supercell. The total energies and forces and optimizations of geometry were carried out using a plane-wave basis set with the projector augmented plane wave method as implemented in the Vienna ab initio simulation package (VASP). ${ }^{13}$ The PW91 form was used for the generalized gradient approximation to exchange and correlation potential. The geometries of clusters were optimized without symmetry constraint using conjugate-gradient algorithm. The energy cutoff and the convergences in energy and force were set to $400 \mathrm{eV}, 10^{-4} \mathrm{eV}$, and $1 \times 10^{-3} \mathrm{eV} / \AA$, respectively. The accuracy of our numerical procedure for carbon, hydrogen, and boron systems was demonstrated in our previous papers. ${ }^{9,10,14,15}$ 


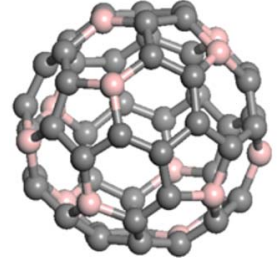

(a)

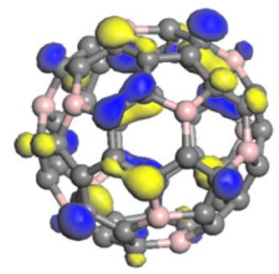

(b)

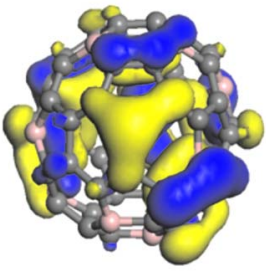

(c)
FIG. 1. (Color online) (a) Geometry, (b) HOMO, and (c) LUMO of $\mathrm{C}_{48} \mathrm{~B}_{12}$.

Past studies showed that due to the curvature and larger $\mathrm{C}-\mathrm{C}$ bond length of the $\mathrm{C}_{60}$ fullerene compared with that of graphite, it is possible to substitutionally dope $\mathrm{C}_{60}$ with $\mathrm{B}$. In particular, Xie et al. ${ }^{12}$ studied the geometries and stability of $\mathrm{C}_{60-n} \mathrm{~B}_{n}$ clusters for $n=1-12$. Experiments performed by Gao et al. ${ }^{16}$ established the existence of $\mathrm{C}_{60-n} \mathrm{~B}_{n}$ clusters $(n$ $=1-6) \cdot C_{48} B_{12}$ was found to have two low lying isomers with $\mathrm{C}_{i}$ and $\mathrm{S}_{6}$ symmetry. ${ }^{12,17}$ The $\mathrm{C}_{i}$ isomer, found by Xie $e t$ $a l .{ }^{12}$ to be the ground state structure, has an ellipsoidal structure with one $\mathrm{B}$ atom per pentagon and two $\mathrm{B}$ atoms preferentially sitting in a hexagon. The distortion of the $\mathrm{C}_{60}$ cage induced by doping is not localized to the neighborhood of each of the dopant atom but rather extends throughout the whole cage. Manaa ${ }^{17}$ showed that a $S_{6}$ isomer, which includes a distribution of $\mathrm{B}$ atoms on the top and bottom of triphenylene-type units and along the equator of $\mathrm{C}_{60}$, is lower in energy than the $\mathrm{C}_{i}$ isomer. Both isomers, however, are electron acceptors. In agreement with the work of Manaa, ${ }^{17}$ we found that the $\mathrm{C}_{i}$ isomer is $0.4 \mathrm{eV}$ higher in energy than the $\mathrm{S}_{6}$ isomer. However, when $12 \mathrm{Li}$ atoms are coated, the complex based on the isomer of $\mathrm{C}_{i}$ symmetry is $0.3 \mathrm{eV}$ lower in energy. So in the following we focus our discussions on the $\mathrm{C}_{i}$ isomer.

The equilibrium geometry of $\mathrm{C}_{i}$ isomer of the $\mathrm{C}_{48} \mathrm{~B}_{12}$ cluster is shown in Fig. 1(a). We find that $\mathrm{B}$ doping decreases the gap between the highest occupied molecular orbital (HOMO) and the lowest unoccupied molecular orbital (LUMO) of $\mathrm{C}_{60}$ from 1.76 to $0.3 \mathrm{eV}$ in $\mathrm{C}_{48} \mathrm{~B}_{12}$, thus making the later more metallic than $\mathrm{C}_{60}$. The $\mathrm{HOMO}$ is mainly contributed by $\mathrm{C}$ atoms [Fig. 1(b)], while the LUMO is from B atoms [Fig. 1(c)]. It is interesting to note ${ }^{12}$ that the changes of average binding energy per atom in $\mathrm{C}_{60-n} \mathrm{~B}_{n}$ are not too big when going from $n=1$ to $n=12$; the corresponding values were found to be $6.77,6.75,6.74,6.73,6.71,6.70,6.67$, $6.66,6.64,6.63,6.62$, and $6.60 \mathrm{eV}$, respectively. We have checked the dynamic stability of $\mathrm{C}_{48} \mathrm{~B}_{12}$ via frequency calculations. We found that there is no imaginary frequency for all the modes, suggesting that $\mathrm{C}_{48} \mathrm{~B}_{12}$ is stable. To further confirm the thermal stability of $\mathrm{C}_{48} \mathrm{~B}_{12}$, we have carried out molecular dynamics simulation by using Nose algorithm ${ }^{18}$ at room temperature $(T=300 \mathrm{~K})$ with 0.4 fs time steps. After 4 ps simulation, we found that the cage geometry of $\mathrm{C}_{48} \mathrm{~B}_{12}$ was still kept.

To determine the equilibrium geometry of $\mathrm{Li}_{12} \mathrm{C}_{48} \mathrm{~B}_{12}$, we studied four isomers shown in Fig. 2. The first choice in Fig. 2(a) was to put $12 \mathrm{Li}$ atoms on top of each $\mathrm{B}$ atom in $\mathrm{C}_{48} \mathrm{~B}_{12}$. Upon optimization, however, the $\mathrm{Li}$ atoms migrated to the top of the pentagon sites, as was found to be the case in $\mathrm{Li}_{12} \mathrm{C}_{60} \cdot{ }^{10}$ To check if clustering of $\mathrm{Li}$ atoms would occur, we used three cluster configurations [Fig. 2(b)-2(d)]. From the optimized structures we see that the clustered configura-
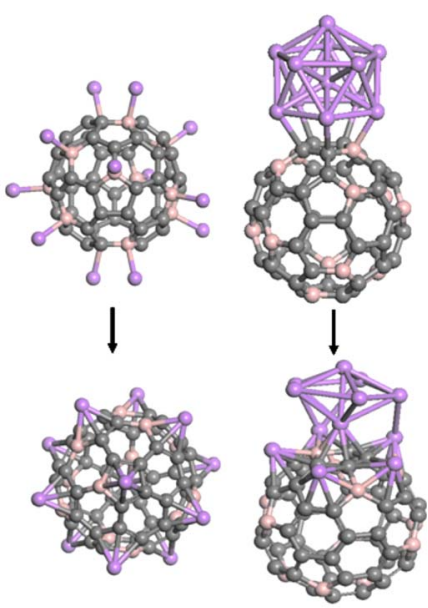

$0.0 \mathrm{eV}$

(a)
$+7.9423 \mathrm{eV}$

(b)

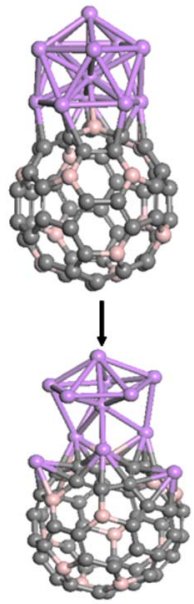

$+8.7178 \mathrm{eV}$

(c)

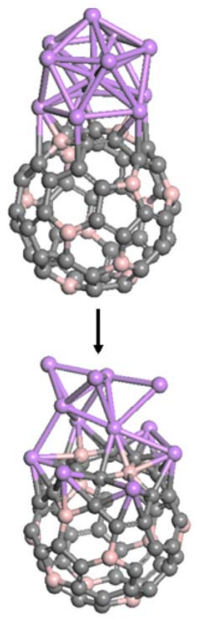

$+7.355 \mathrm{eV}$

(d)
FIG. 2. (Color online) The initial (the upper panel) and the optimized geometries (the lower panel) of $\mathrm{Li}_{12} \mathrm{C}_{48} \mathrm{~B}_{12}$ complexes. The energies are measured with respect to the ground state in (a).

tions are higher in energy by $7.355,7.942$, and $8.718 \mathrm{eV}$, respectively, as compared to the isolated configuration [Fig. 2(a)]. It is important to note that in $\mathrm{Li}_{12} \mathrm{C}_{60}$ the clustered configuration was only $2.2 \mathrm{eV}$ higher in energy than the isolated configuration. Thus substituting $\mathrm{C}$ by $\mathrm{B}$ in the heterofullerene further enhances the stability of the isolated configuration.

$\mathrm{Li}_{12} \mathrm{C}_{48} \mathrm{~B}_{12}$ and $\mathrm{Li}_{12} \mathrm{C}_{60}$ also have quite different electronic structures as shown in Fig. 3. For example, the LUMO of $\mathrm{Li}_{12} \mathrm{C}_{60}$ is mainly from the coating layer of $\mathrm{Li}$, while it is from $\mathrm{C}$ and $\mathrm{B}$ in $\mathrm{Li}_{12} \mathrm{C}_{48} \mathrm{~B}_{12}$.

Next we have studied the absorption of hydrogen molecules on the $\mathrm{Li}_{12} \mathrm{C}_{48} \mathrm{~B}_{12}$ heterofullerene. We began by plac-

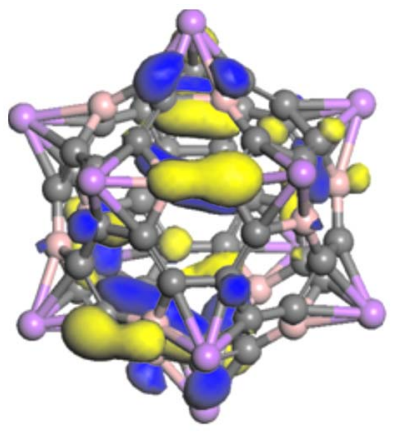

(a)

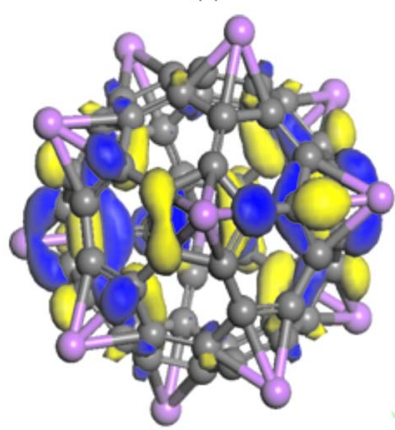

(c)

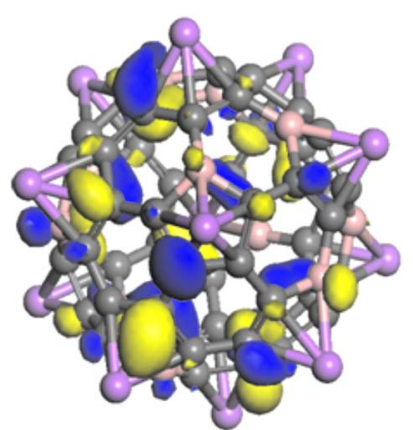

(b)

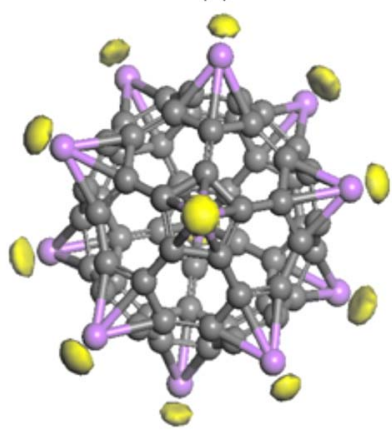

(d)
FIG. 3. (Color online) (a) HOMO and (b) LUMO of $\mathrm{Li}_{12} \mathrm{C}_{48} \mathrm{~B}_{12}$ and (c) HOMO and (d) LUMO of $\mathrm{Ii}_{\mathrm{C}} \mathrm{C}$ 


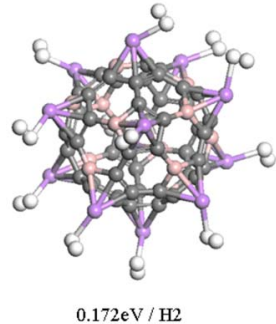

(a)

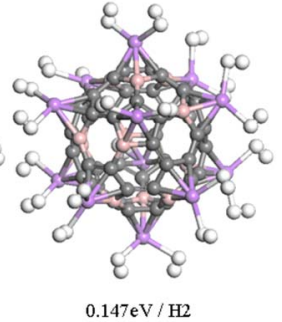

(b)

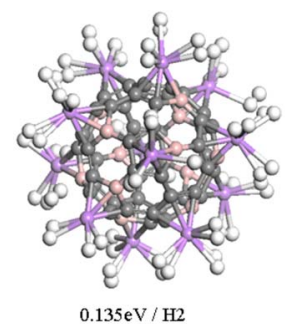

(c)
FIG. 4. (Color online) Hydrogen absorption in $x \mathrm{H}_{2}-\mathrm{Li}_{12} \mathrm{C}_{48} \mathrm{~B}_{12}$ with (a) $x$ $=12$, (b) 24 , and (c) 36 .

ing one $\mathrm{H}_{2}$ molecule on the top site of each Li [Fig. 4(a)] and then optimized the geometry without any symmetry restriction. We found that hydrogen is bound molecularly with a binding energy of $0.172 \mathrm{eV} / \mathrm{H}_{2}$ but with a slightly stretched $\mathrm{H}-\mathrm{H}$ bond length of $0.761 \AA$. The distance between $\mathrm{Li}$ and the nearest $\mathrm{H}$ atom is $1.99 \AA$. When we increased the number of $\mathrm{H}_{2}$ molecules to 24 by placing two $\mathrm{H}_{2}$ molecules on each $\mathrm{Li}$ site, the binding energy decreased to $0.147 \mathrm{eV} / \mathrm{H}_{2}$, and the bond length of $\mathrm{H}_{2}$ became $0.757 \AA$. The nearest distance of $\mathrm{H}$ from the $\mathrm{Li}$ site increased to $2.10 \AA$. With three $\mathrm{H}_{2}$ molecules placed on each $\mathrm{Li}$ site, i.e., a total of $36 \mathrm{H}_{2} \mathrm{~mol}-$ ecules, the binding energy decreased to $0.135 \mathrm{eV} / \mathrm{H}_{2}$, with a corresponding decrease in $\mathrm{H}-\mathrm{H}$ bond length to $0.753 \AA$. The nearest distance of $\mathrm{H}$ from the $\mathrm{Li}$ site increased to $2.20 \AA$. In Table I, we summarize these results. With $36 \mathrm{H}_{2}$ molecules adsorbed on a $\mathrm{Li}_{12} \mathrm{C}_{48} \mathrm{~B}_{12}$ cluster, the gravimetric density reaches 9 wt \% and average binding energy per $\mathrm{H}_{2}$ molecule is $0.135 \mathrm{eV}$. This is almost a factor of 2 larger than the corresponding average binding energy per $\mathrm{H}_{2}$ in $\mathrm{Li}_{12} \mathrm{C}_{60}$. Thus, we have shown that $\mathrm{B}$ doping of $\mathrm{C}_{60}$, namely, $\mathrm{C}_{48} \mathrm{~B}_{12}$ substantially improves the hydrogen binding energy and hence improves its performance as a hydrogen storage material. This improvement is attributed to the electron acceptor property of $\mathrm{C}_{48} \mathrm{~B}_{12}$.

We have also investigated the hydrogen storage ability of $\mathrm{Li}$ coated $\mathrm{C}_{48} \mathrm{~N}_{12}$, which has been synthesized experimentally. ${ }^{19}$ Unfortunately $\mathrm{C}_{48} \mathrm{~N}_{12}$ is not a good candidate. Since $\mathrm{N}$ atom has one more valence electron than $\mathrm{C}$, $\mathrm{C}_{48} \mathrm{~N}_{12}$ is an electron-rich complex and behaves like a donor.

TABLE I. Number of $\mathrm{H}_{2}$ molecules $x$, binding energy $E_{b}\left(\mathrm{in} \mathrm{eV} / \mathrm{H}_{2}\right)$, bond length of $\mathrm{H}_{2} R_{\mathrm{H} 2}$ (in angstrom), the distance between $\mathrm{H}_{2}$ and $\mathrm{Li}$ ions $R_{\mathrm{Li}-\mathrm{H}_{2}}$ (in angstom), and the weight percentage wt $\%$ for $x \mathrm{H}_{2}-\mathrm{C}_{48} \mathrm{~B}_{12} \mathrm{Li}_{12} \quad(x=12$, 24 , and 36).

\begin{tabular}{ccccc}
\hline \hline$x$ & $E_{b}$ & $R_{\mathrm{H}_{2}}$ & $R_{\mathrm{Li}-\mathrm{H} 2}$ & wt \% \\
\hline 12 & 0.172 & 0.761 & 1.990 & 3 \\
24 & 0.147 & 0.757 & 2.100 & 6 \\
36 & 0.135 & 0.753 & 2.200 & 9 \\
\hline \hline
\end{tabular}

Since $\mathrm{Li}$ atoms prefer to donate their $2 s$ electrons, the structure of $\mathrm{Li}_{12} \mathrm{C}_{48} \mathrm{~N}_{12}$ is very different in geometry and property. In fact, we find that these $12 \mathrm{Li}$ atoms prefer to cluster instead of remaining isolated. Consequently, the ability of $\mathrm{Li}_{12} \mathrm{C}_{48} \mathrm{~N}_{12}$ is severely undermined.

In summary, based on gradient corrected density functional theory, we have shown that Li decorated boron doped $\mathrm{C}_{60}$ heterofullerene has several advantages over Li decorated $\mathrm{C}_{60}$ fullerene for storing hydrogen. (1) $\mathrm{Li}$ atoms in $\mathrm{Li}_{12} \mathrm{C}_{48} \mathrm{~B}_{12}$, like that in $\mathrm{Li}_{12} \mathrm{C}_{60}$, do not cluster. In addition, the isolated state in the former is energetically far more stable than in the later. (2) $\mathrm{B}$ doping of $\mathrm{C}_{60}$ improves the weight percentage of stored hydrogen as $B$ is lighter than $C$. (3) Since $B$ has one electron less than $C$, the resulted $C_{48} B_{12}$ heterofullerene is electron-deficient and behaves like an electron acceptor. This makes it possible for the $\mathrm{Li}$ atoms to freely donate their $2 s$ electrons to the heterofullerene, thereby remaining in a positively charged state. (4) Up to three $\mathrm{H}_{2}$ molecules per $\mathrm{Li}$ atom can be attached to the $\mathrm{Li}_{12} \mathrm{C}_{48} \mathrm{~B}_{12}$ heterofullerene leading to a gravimetric density of 9 wt \%. (5) The average binding energy of $\mathrm{H}_{2}$ molecules lies between physisorption and chemisorption energies and is almost twice as large as that in $\mathrm{Li}_{12} \mathrm{C}_{60}$, lending the possibility that $\mathrm{B}$ doped $\mathrm{C}_{60}$ fullerenes may be suitable as a hydrogen storage material in ambient temperature. ${ }^{1}$

This work is partially supported by grants from the National Natural Science Foundation of China (Grant Nos. NSFC-10744006 and NSFC-10874007) and from the U.S. Department of Energy.

${ }^{1}$ S. K. Bhatia and A. L. Myers, Langmuir 22, 1688 (2006).

${ }^{2}$ K. Komatsu, M. Murata, and Y. Murata, Science 238, 307 (2005).

${ }^{3}$ O. V. Pupysheva, A. A. Farajian, and B. I. Yakobson, Nano Lett. 8, 767 (2008).

${ }^{4}$ M. Yoon, S. Yang, E. Wang, and Z. Zhang, Nano Lett. 7, 2578 (2007).

${ }^{5}$ K. R. S. Chandrakumar and S. K. Ghosh, Nano Lett. 8, 13 (2008).

${ }^{6}$ O. Byl, J. Liu, Y. Wang, W.-L. Yim, J. K. Johnson, and J. T. Yates, J. Am. Chem. Soc. 128, 12090 (2006).

${ }^{7}$ Y. Zhao, Y.-H. Kim, A. C. Dillon, M. J. Heben, and S. B. Zhang, Phys. Rev. Lett. 94, 155504 (2005).

${ }^{8}$ T. Yildirim and S. Ciraci, Phys. Rev. Lett. 94, 175501 (2005).

${ }^{9}$ Q. Sun, Q. Wang, P. Jena, and Y. Kawazoe, J. Am. Chem. Soc. 127, 14582 (2005).

${ }^{10}$ Q. Sun, P. Jena, Q. Wang, and M. Marquez, J. Am. Chem. Soc. 128, 9742 (2006).

${ }^{11}$ B. K. Rao and P. Jena, Europhys. Lett. 20, 307 (1992).

${ }^{12}$ R. H. Xie, G. W. Bryant, J. Zhao, V. H. Smith, A. D. Carlo, and A Pecchia, Phys. Rev. Lett. 90, 206602 (2003).

${ }^{13}$ G. Kresse and J. Hurthmüller, Phys. Rev. B 54, 11169 (1996).

${ }^{14}$ Q. Sun, Q. Wang, P. Jena, B. K. Rao, and Y. Kawazoe, Phys. Rev. Lett. 90, 135503 (2003).

${ }^{15}$ Q. Sun, Q. Wang, and P. Jena, Nano Lett. 5, 1273 (2005).

${ }^{16}$ T. Guo, C. Jin, and R. E. Smalley, J. Phys. Chem. 95, 4948 (1991).

${ }^{17}$ M. Riad Manaa, Chem. Phys. Lett. 382, 194 (2003).

${ }^{18}$ S. Nose, J. Chem. Phys. 81, 511 (1984).

${ }^{19}$ B. Brena and Y. Luo, J. Chem. Phys. 119, 7139 (2003). 